\title{
Fusiform Activation to Animals is Driven by the Process, Not the Stimulus
}

\author{
Timothy T. Rogers ${ }^{1,2}$, Julia Hocking ${ }^{3}$, Andrea Mechelli ${ }^{3}$, \\ Karalyn Patterson ${ }^{1}$, and Cathy Price ${ }^{3}$
}

\begin{abstract}
Previous studies have found that the lateral posterior fusiform gyri respond more robustly to pictures of animals than pictures of manmade objects and suggested that these regions encode the visual properties characteristic of animals. We suggest that such effects actually reflect processing demands arising when items with similar representations must be finely discriminated. In a positron emission tomography (PET) study of category verification with colored photographs of animals and vehicles, there was robust animal-specific activation in the lateral posterior fusiform gyri when stimuli were categorized at an intermediate level of specificity (e.g.,
\end{abstract}

\section{INTRODUCTION}

Several studies have been published in recent years designed to illuminate the neural underpinnings of human conceptual knowledge about objects and their properties. Among the more provocative findings in this literature are reports of cortical regions that seem to respond more robustly for some categories of objects than for others. For instance, in an fMRI study by Chao, Haxby, and Martin (1999), the lateral aspects of the left and right posterior fusiform gyri were activated more strongly in semantic tasks involving pictures of animals or faces compared with tools or houses, and a second region situated more medially revealed the reverse pattern. A great many other studies have also reported apparent category-specific patterns of responding within the temporal cortex and elsewhere (for recent reviews, see Thompson-Schill, 2003; Joseph, 2001; Martin \& Chao, 2001).

Such results are provocative in part because there is little agreement as to what they signify about the functional organization of the neural system that underlies concept knowledge. Most often, category-specific patterns of responding are interpreted as reflecting some-

\footnotetext{
${ }^{1} \mathrm{MRC}$ Cognition and Brain Sciences, Cambridge, ${ }^{2}$ University of Wisconsin-Madison, ${ }^{3}$ Wellcome Department of Imaging Neuroscience, London
}

dog or car). However, when the same photographs were categorized at a more specific level (e.g., Labrador or BMW), these regions responded equally strongly to animals and vehicles. We conclude that the lateral posterior fusiform does not encode domain-specific representations of animals or visual properties characteristic of animals. Instead, these regions are strongly activated whenever an item must be discriminated from many close visual or semantic competitors. Apparent category effects arise because, at an intermediate level of specificity, animals have more visual and semantic competitors than do artifacts. thing about the kind of information processed by a given region of cortex. For instance, domain-specific representations might be parcellated within a semantic system such that different categories of objects are processed by anatomically distinct cortical regions (Caramazza \& Mahon, 2003; Caramazza, 1998; Caramazza \& Shelton, 1998). Alternatively, a given cortical region might respond selectively to certain visual attributes, which happen to be more characteristic of one category than another (Martin \& Chao, 2001). In either case, the category-specific pattern is understood to be driven by the properties of the stimulus items themselveseither their semantic category membership or their characteristic visual properties-and thus to reveal something about the neuroanatomical organization of conceptual representations.

An alternative hypothesis is that apparent category specificity actually reflects processing demands that are jointly determined by representational structure, and by the particular task being performed (Bukach, Bub, Masson, \& Lindsay, 2004; Price, Noppeney, Phillips, \& Devlin, 2003; Gale, Done, \& Frank, 2001; Gauthier, 2000; Gorno-Tempini, Cipolotti, \& Price, 2000; Tarr \& Gauthier, 2000; Tyler, Moss, Durrant-Peatfield, \& Levy, 2000; Moore \& Price, 1999; Gaffan \& Heywood, 1993). Specifically, the fusiform may be strongly activated by tasks that require participants to discriminate an object from many similar visual or semantic competitors (Joseph \& Gathers, 2003; Price et al., 2003). On this view, differential activation in 
response to animals might occur because, at an intermediate level of specificity, animal concepts tend to have many visual and semantic properties in common, whereas artifact concepts do not. For example, pigs, goats, horses, dogs, and other four-legged mammals have similar overall shapes (Rosch, Mervis, Gray, Johnson, \& Boyes-Braem, 1976), share many of the same parts (Tversky \& Hemenway, 1984), and have many functional and behavioral attributes in common (Garrard, Lambon Ralph, Hodges, \& Patterson, 2001), despite having different basic-level names. By contrast, most artifact categories tend to be more loosely structured: Different kinds of tools can have quite different shapes, parts, and functions, and the same can be said of household objects, vehicles, and furniture (Rogers et al., 2004; McRae \& Cree, 2002; Garrard et al., 2001; Tyler et al., 2000). Thus, when participants must name or otherwise identify stimuli at an intermediate level of specificity, there may be a discrepancy between domains in the ease with which items are discerned from their visual and semantic neighbors.

In visual semantic tasks, patterns of fusiform activation are known to be strongly affected by the demands of perceptual differentiation (see Joseph \& Gathers, 2003), with fusiform activation increasing as the perceptual discrimination gets harder. In some cases, such sensitivity to perceptual discriminability can produce apparent category effects. For instance, the fusiform face area (FFA) is a region in the posterior fusiform that responds more strongly to faces than to other natural objects in a range of different tasks. Kanwisher and colleagues have suggested that the FFA constitutes a cortical module specialized through evolution for the recognition and representation of faces (Grill-Spector, Knouf, \& Kanwisher, 2004; Kanwisher, Stanley, \& Harris, 1999; Kanwisher, McDermott, \& Chun, 1997). Gauthier and colleagues, however, have shown that the FFA comes to respond to (perceptually similar) objects after participants have learned to discriminate themsuggesting that activation in this area is modulated primarily by expertise and by demands for perceptual differentiation (Gauthier, Skudlarski, Gore, \& Anderson, 2000; Tarr \& Gauthier, 2000; Gauthier, Tarr, Anderson, Skudlarski, \& Gore, 1999). It is possible that the apparent category effects reported by Chao et al. (1999) have a similar explanation: Apparent animal-related activity in the posterior fusiform may arise largely because this region must work harder to differentiate representations of individual animals than individual artifacts (Gale et al., 2001; Gaffan \& Heywood, 1993; Humphreys, Riddoch, \& Quinlan, 1988).

When participants must identify items at a specific level, such "structural" differences between animal and artifact domains are attenuated. For both domains, specific categorization tasks-determining that a particular dog is a Labrador retriever or that a particular car is a BMW-demand that the stimulus representation be discerned from many similar competitors (i.e., other familiar breeds of dog or makes of car). The processing demands entailed by specific categorization tasks are thus likely to be similar for animals and artifacts. This prediction provides a simple means of assessing whether the observations of animal-related activity in the posterior fusiform reported by Chao et al. (1999) truly reveal the kind of information encoded in this region of cortex or whether such patterns reflect the processing demands that derive from representational structure. If category-specific effects arise because the lateral posterior fusiform encodes a particular kind of information-either domain-specific representations of animals or visual properties characteristic of animals-then such effects should be observed there regardless of the specificity with which items are identified. By contrast, if animal-related activity actually reflects processing demands deriving from representational structure, then the category-specific pattern should be observed when items are categorized at an intermediate level of specificity (where structural differences between animal and artifact domains are greatest). However, such effects should be attenuated or eliminated when the same items are categorized at a more specific level.

We tested this idea using positron emission tomography (PET) in a category-verification paradigm, in which participants categorized color photographs of real objects at three different levels of specificity. On each trial, participants viewed either a very general category name (e.g., animal or vehicle), an intermediate-level name (e.g., bird or boat), or a specific name (e.g., robin or ferry), followed by a color photograph of a common object (a particular animal or vehicle). The task was to determine whether the object matched the category label. Trials were organized into blocks, with one block equating to one scan in the PET session, so that (a) within block, stimuli were categorized at the same level of specificity (general, intermediate, or specific), (b) across blocks, exactly the same set of photographs were viewed in each specificity condition, (c) the same number of different category labels appeared in every block, (d) all blocks had an equivalent number of trials, and (e) intermediate and specific blocks were further organized by semantic domain, with participants viewing only animals in some blocks and only vehicles in others (animal and vehicle pictures were intermixed in the general condition). This design thus allowed us to investigate the specificity of the categorization task, semantic category effects, and the interaction of these factors, using the same stimulus materials and the same task across experimental conditions.

In addition to the experimental task, the design of the PET study included a baseline task in which participants viewed the word left or right on each trial, followed by a scrambled color photograph, and were 
instructed to press the left or right response button accordingly.

\section{RESULTS}

\section{Behavioral Pilot}

The experimental task was piloted outside of the scanner on 12 right-handed men (aged 19-39 years, mean age of 25 years) to assess the relative speed and accuracy with which healthy controls could classify the various animals and vehicles at the different levels of specificity. Means and standard errors for the reaction times (RTs) are shown in Figure 1. Repeated measures analyses of variances (ANOVAs) showed reliable differences in RTs across conditions, $F(2,22)=8.3, p<.002$, with Bonferroni-corrected post hoc contrasts showing that the controls were faster to classify these materials at the intermediate level of specificity compared with more general $(p<.03$, post hoc) or specific $(p<.001$, post hoc) levels, consistent with the large literature on basiclevel advantages (e.g., Rosch et al., 1976). RTs were not reliably different, however, for classification at the general and specific levels, nor were there any reliable differences across conditions in accuracy, $F(2,22)=$ $0.91, p=n s$, with controls scoring better than $95 \%$ correct in all conditions. Most importantly for the current study, participants were equally fast and accurate to verify category membership for animals and vehicles [speed: $F(1,11)=3.38, p=n s$; accuracy: $F(1,11)=0.31$, $p=n s]$ in both intermediate and specific conditions, with no reliable interaction between semantic category and task condition [speed: $F(1,11)=0.24, p=n s$; accuracy: $F(1,11)=0.25, p=n s]$. Thus, although participants generally find the intermediate classification easier, there is no indication of a semantic category effect in the ease of classification at either the intermediate or specific level.

\section{Positron Emission Tomography Results}

We report the simple contrast of the experimental to baseline scans; in the current study, however, we are primarily interested in main effects of domain (animal vs. vehicle) and the interaction of this effect with task condition (specific, intermediate, and general) in the fusiform areas reported to show greater activation for animals relative to tools in the studies by Martin and Chao (2001) and Chao et al. (1999). Thus, we report wholebrain analyses thresholded at $p<.05$, corrected for multiple comparisons, as well as region of interest (ROI) analyses based on the coordinates for animal-related fusiform activation reported previously by Chao et al. (1999) during their silent naming task $(x, y$, and $z$ coordinates: right fusiform, $37,-55$, and -21 ; left fusiform, $-37,-55$, and -20 ). The ROI had a radius of $12 \mathrm{~mm}$. Note that domain effects and interactions could only be assessed for the specific and intermediate scanning blocks, because items from both domains were intermixed within scan in the general condition. The effects of interest were estimated according to the general linear model at each voxel.

The contrast of all experimental scans to the baseline scans revealed activations reliable at a corrected level of significance of $p<.05$ in the bilateral occipito-temporal cortex and a broad expanse of the posterior and anterior fusiform. The peak coordinates according to the atlas of Talairach and Tournoux (1988) are shown in Table 1. These results are commensurate with the literature on object processing: Occipito-temporal activation is likely a result of the fact that the real photographs in the
Figure 1. Mean RTs to verify category membership in the behavioral pilot study. The left panel shows the means for the three specificity conditions, collapsed across animal and vehicle blocks. The right panel shows the means plotted separately for animal and artifact blocks in the specific and intermediate conditions. Error bars indicate standard errors.

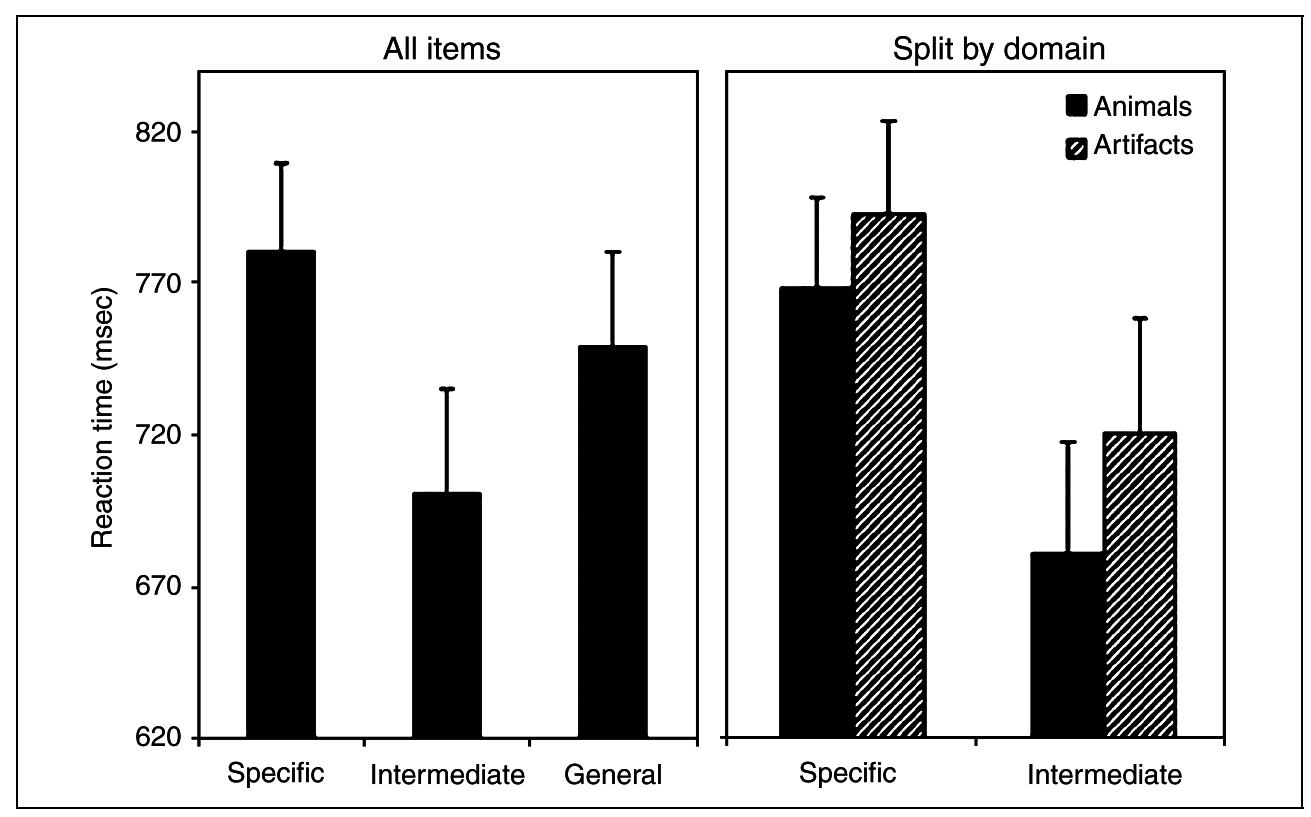


experimental conditions are visually more well formed and were processed more deeply than the scrambled photographs in the baseline condition. Posterior fusiform activation is commonly reported in object recognition tasks relative to nonsemantic visual baseline conditions (Malach, Levy, \& Hasson, 2002) and more anterior activation within the fusiform is commonly reported in semantic tasks (e.g., Mummery, Patterson, Hodges, \& Price, 1998).

Figure 2A shows the contrast of all experimental scans involving animals (collapsed across condition) to those involving vehicles. Activation peaks were observed in the lateral aspect of the posterior fusiform on the right $(x=48, y=-54, z=-22, Z=5.0, p<.05$, corrected for the whole brain) with a corresponding trend on the left $(x=-40, y=-68, z=-14, Z=3.6$, $p<.6$, corrected for the whole brain). When the analysis was focused on our ROIs, two activation peaks were apparent in the left hemisphere, one in posterior fusiform $(x=-40, y=-64, z=-16, Z=3.5, p<.01$, corrected for $12 \mathrm{~mm}$ ) and one more anterior that did not reach significance $(x=-38, y=-48, z=-24, Z=$ $2.5, p=0.18$, corrected for $12 \mathrm{~mm}$ ). Only one peak was apparent in the right ROI, and this was statistically reliable ( $x=48, y=-54, z=-22, Z=5.0, p<.01)$.

All three peaks correspond well to the animal-related activations in the posterior fusiform described by Chao et al. (1999), who report peaks ranging from $x=-35$ to $-40, y=-55$ to $-59, z=-10$ to -21 in the left and $x=37$ to $41, y=-52$ to $-56, z=-12$ to -21 in the right. Thus, the current data replicate Chao et al.'s (1999) finding of apparent animal-related activation in the lateral aspect of the posterior fusiform gyrus. The reverse contrast (vehicles-animals, collapsed across experimental condition) revealed no activations reliable at a corrected level of significance for the wholebrain analysis.

The main effect of category described above interacted with level of specificity. Within our ROI, an interaction was observed in the right fusiform at $(x=48, y=$ $-48, z=-22, Z=3.8, p=0.016$, corrected for $12 \mathrm{~mm}$ ). A corresponding trend approached significance in the left fusiform at the more anterior peak $(x=-30, y=$ $-50, z=-12, Z=2.5, p=0.18$, corrected for $12 \mathrm{~mm}$ ),

Table 1. Peak Coordinates in Bilateral Occipito-Temporal Cortices for All Semantic Tasks Relative to Baseline

\begin{tabular}{|c|c|c|c|c|c|c|c|}
\hline \multicolumn{4}{|c|}{ Left } & \multicolumn{4}{|c|}{ Right } \\
\hline$x$ & $y$ & $z$ & $Z$ & $x$ & $y$ & $z$ & $Z$ \\
\hline-42 & -74 & -16 & (5.9) & 48 & -78 & -12 & (7.6) \\
\hline-32 & -90 & 4 & (6.9) & 38 & -88 & 4 & (4.6) \\
\hline-38 & -56 & -18 & $(5.6)$ & 38 & -60 & -18 & (5.8) \\
\hline-28 & -38 & -16 & (5.6) & 26 & -28 & -20 & (5.2) \\
\hline
\end{tabular}

but not at the more posterior peak $(x=-46, y=-62$, $z=-16, Z=1.5, p=n s$, corrected for $12 \mathrm{~mm}$ ). The peak of the Category $\times$ Task interaction is shown in Figure $2 \mathrm{~B}$.

The interaction is the result of (a) higher activation for animals than vehicles during intermediate categorization but not during specific categorization and (b) higher activation for specific than intermediate categorization on vehicles but not animals (see Table 2). Figure 2C shows mean-centered effect sizes in the left and right fusiform, in the baseline condition and the five experimental conditions. Corrected post hoc contrasts showed that considering the intermediate blocks only, the effect of category was significant at the right peak $(x=48, y=-52, z=-22, Z=5.1, p<.001)$ and the left anterior peak $(x=-30, y=-54, z=-12, Z=2.93$, $p<.05)$, whereas a strong trend in the same direction was observed for the more posterior left peak $(x=-46$, $y=-62, z=-16, Z=2.8, p<.06$ ). Considering just the specific blocks, the effect of category did not approach significance at any of the three peaks (right: $x=46, y=-58, z=-18, Z=2.1, p=n s$; left anterior: $x=-34, y=-54, z=-16, Z=1.2, p=n s$; left posterior: $x=-38, y=-64, z=-16, Z=2.4, p=n s$ ). Thus, category effects were observed when participants categorized objects at an intermediate level of specificity, with animals strongly activating the lateral posterior fusiform relative both to vehicles and to baseline. When the same items were categorized at the specific level, however, no significant effect of category was observed: The same regions were robustly activated for both animals and vehicles relative to baseline. Finally, the figure indicates that these regions were also activated in the general condition relative to baseline.

\section{DISCUSSION}

Robust activation in the posterior and lateral aspects of the fusiform gyrus, bilaterally, was observed in a category-verification task involving animals and vehicles. When participants categorized objects at an intermediate level, this activation appeared to be specific to animals. For categorization of the same objects at a more specific level, however, the region responded strongly to both animals and vehicles, and no significant domain effect was observed. The same region also responded when participants classified the same items at a general level of specificity. An analysis of behavioral data showed that animals and vehicles were classified with equal speed and accuracy within the intermediate and specific conditions, so that the effect of category, and its interaction with task condition, cannot be explained with reference to the relative difficulty of classifying animals versus vehicles in the different conditions. Nor can the task effects be explained with reference to properties of the stimulus items, because the same 48 items were viewed in all three task conditions. Thus, we 
Figure 2. (A) Contrast of scans involving animals to those involving vehicles, collapsed across specific and intermediate conditions, with an uncorrected threshold of $p<.01$ to show the full extent of activation. The analysis reveals greater activation for animals relative to vehicles in the lateral posterior fusiform on both sides, with peaks in Talairach space at $-40,-68$, and -14 on the left and 48, -54 , and -22 on the right. (B) Interaction of domain and task condition for specific and intermediate scans, thresholded at an uncorrected level of $p<.05$ to show the full extent of activation. The peaks of the interaction term in Talairach coordinates are -32 , -52 , and -14 in the left and $44,-50$, and -22 in the right. (C,D) Mean-centered effect sizes for all conditions, at the peaks of the interaction term in the left and right posterior lateral fusiform gyri. The interaction arises from greater activation for animals than vehicles in the intermediate condition, but not the specific condition.

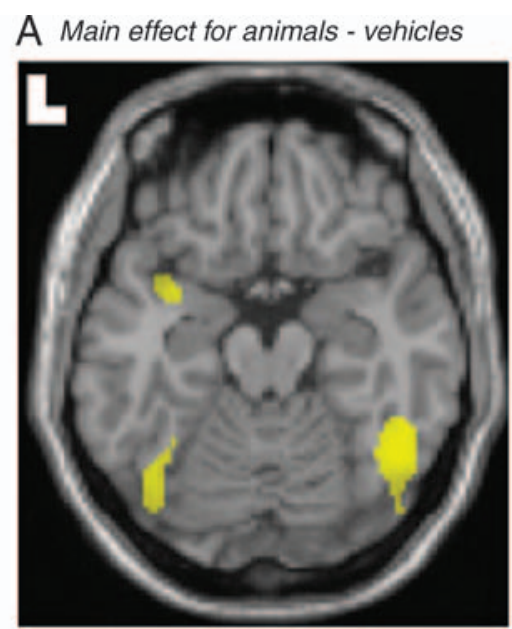

$Z=-16$

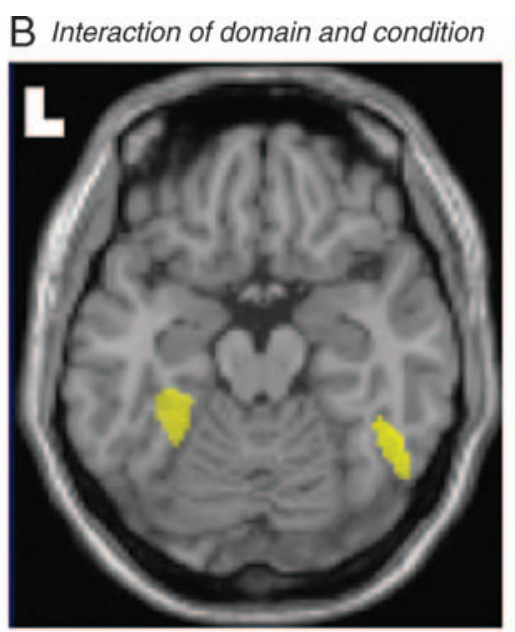

$Z=-18$

\section{Mean-centered effect size for peaks of interaction term on both sides}
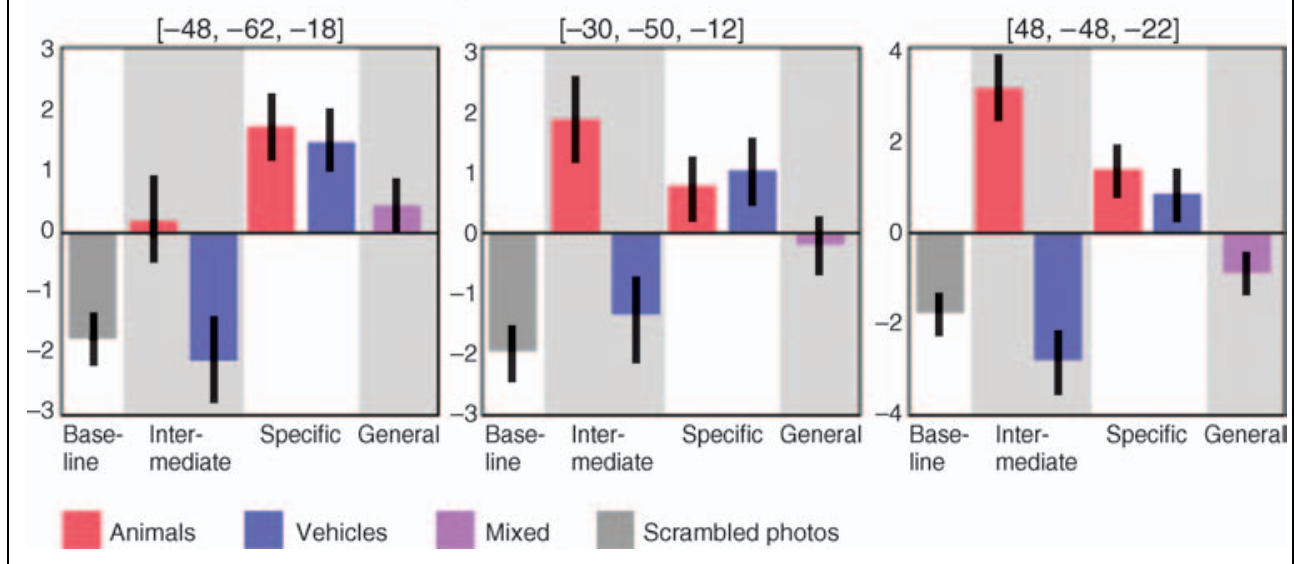

have shown that reliable domain effects may or may not be observed in the same semantic task, for the same stimulus items, depending upon the level of specificity at which the items must be identified.

The results are important for two reasons. First, they provide a replication of Chao et al.'s (1999) basic finding of animal-related activation in the lateral posterior fusiform. Such effects have been replicated previously (e.g., Price et al., 2003), but with less than perfect consistency. In a meta-analysis of functional imaging studies of conceptual knowledge, Joseph (2001) reported a general tendency for tasks involving animals to activate lateral fusiform regions somewhat more strongly than artifacts; however these domain differences were present in less than half of the surveyed studies that might have detected them, and moreover, category effects accounted for less variance overall than did task effects in the meta-analysis. Tyler and Moss (2001) have emphasized that there is more similarity than difference in the cortical networks activated by animals and artifacts

Table 2. Location and $Z$ Scores for Peaks of Interaction in Left and Right Fusiform

\begin{tabular}{|c|c|c|c|c|c|c|c|}
\hline & \multicolumn{4}{|c|}{ Location } & \multicolumn{3}{|c|}{$Z$ Scores for Post Hoc Simple Main Effects } \\
\hline & $x$ & $y$ & $z$ & $Z$ & $\begin{array}{l}\text { Animal (Int) } \\
\text { - Vebicle (Int) }\end{array}$ & $\begin{array}{l}\text { Animal (Spec) } \\
\text { - Vebicle (Spec) }\end{array}$ & $\begin{array}{c}\text { Vebicle (Int) } \\
- \text { Vebicle (Spec) }\end{array}$ \\
\hline Right & 48 & -54 & -22 & 3.8 & 5.0 & 2.1 & 3.6 \\
\hline Left anterior & -30 & -50 & -12 & 2.5 & 2.9 & 1.2 & 2.5 \\
\hline Left posterior & -46 & -62 & -16 & 1.5 & 2.8 & 2.4 & 3.7 \\
\hline
\end{tabular}

Note: $I n t=$ intermediate level; Spec $=$ specific level. 
in their own PET and fMRI studies of semantic task performance. The main effect of category observed in the current study bolsters the reliability of the basic phenomenon, and indicates that animal-related activation in the fusiform may be observed in contrasts involving vehicles, in addition to contrasts involving tools and houses (Price et al., 2003; Chao et al., 1999). Furthermore, the results demonstrate that such category effects do not simply reflect overall task difficulty, as participants were equally fast and accurate to classify animals and vehicles in this experiment.

Second and more important, the current study suggests one reason why category effects are only sporadically observed in these studies. Specifically, the data suggest that category-specific responding in the lateral fusiform does not indicate that this region encodes domain-specific representations of animals or visual attributes characteristic of animals. Instead, such patterns appear to reflect the processing demands of the particular task being performed, which may in turn arise from the similarity structure of the representations coded in this region of cortex (Price et al., 2003; Joseph, 2001; Tarr \& Gauthier, 2000; Humphreys, Riddoch, \& Price, 1997). When the task requires participants to discriminate items with similar visual or semantic representations, the posterior fusiform is likely to be activated. As several studies have indicated, animals with different basic-level names (including birds and dogs) are likely to share many visual and semantic properties, whereas artifacts with different basic-level names (including different kinds of vehicle) are more distinct (Rogers et al., 2004; McRae \& Cree, 2002; Garrard et al., 2001; Tyler \& Moss, 2001). Hence, naming tasks, and other tasks that require participants to identify animals at an intermediate level of specificity, are likely to produce category-specific patterns of activation in the lateral posterior fusiform. Our results indicate, however, that when the differentiation demands are equated for animal and artifact categories, no such pattern will be observed.

Although the studies of verbal attribute listing and visual-feature overlap cited above all suggest that animals from different intermediate categories have more overlapping properties than do artifacts, it is worth noting that, in our behavioral study, animals were categorized as rapidly and accurately as artifacts at both intermediate and specific levels. This observation is important, as it allows us to decouple differentiation demands from overall task difficulty: The category effects observed in the intermediate condition cannot have arisen simply because animals were more difficult to classify in some absolute sense. However, this raises a further puzzle: If animals are more difficult to discriminate at a visual or conceptual level, why are participants not slower to categorize the animals?

A complete answer to this question is the topic of a current modeling project that is beyond the scope of this discussion. By way of a partial answer, however, we suggest that the processing required for word-picture matching is distributed across cortical regions that perform different computations in parallel. For instance, the task probably recruits various different regions that (a) activate the appropriate orthographic and/or phonological word forms, (b) activate appropriate visual representations of the object, and (c) compute the crossmodal mapping between the object form and the word form. Processing in this network occurs in parallel and is interactive, so that the settling process occurring in regions that compute phonological representations is partially constrained by concurrent settling processes in the cross-modal and visual regions. On this view, RTs result from the settling of the entire network: Only when visual and word representations are both sufficiently well resolved can the participant respond accurately. Settling processes within individual components of the network-for instance, within the subnetwork that computes visual representations-only partially contribute to the full network settling time, and hence may or may not coincide with the total time to settle across the whole network. There is also, presumably, a significant component of the measured response time that is attributable to generating the key-press response. Our proposal is that, although one part of this mosaic of processes contributing to the final measured RT may take longer to complete (and correspondingly engender more fusiform activation) for animals than vehicles at the intermediate level, this component may be "swamped" by others in the full cascade of processes and thus not be reflected in the single RT (see e.g., Monsell, Doyle, \& Haggard, 1989).

\section{Relation to Other Work}

Several studies have reported category-specific patterns of responding within the temporal lobes and elsewhere. Perhaps the best-known studies of specialization within the fusiform are those concerning the perception and representation of faces. As noted in the Introduction, the pioneering work of Kanwisher and colleagues found a region in the middle fusiform gyrus, which they dubbed the fusiform face area, that responded more strongly to visual depictions of faces than to other visual stimuli across a range of different tasks (Kanwisher, 2000; Kanwisher, Stanley, \& Harris, 1999; Kanwisher, McDermott, \& Chun, 1997). One interpretation of these findings is that they reveal a "content-based" organization of visual cortex, in which different anatomical regions are specialized to represent some kinds of visual stimuli and not others. However, Kanwisher's work has spurred a considerable volume of research that has not altogether supported a strict view of functional specialization. For instance, the influential work of Gauthier and colleagues has shown that the FFA responds to objects other than faces (Gauthier, 2000; see also Joseph \& 
Gathers, 2003), that it responds more to items within a domain of expertise than to items outside the expert domain (Gauthier, Skudlarski, et al., 2000; Tarr \& Gauthier, 2000), and that it responds increasingly as novices learn to name and categorize visually similar nonsense objects (Gauthier, Tarr, et al., 1999). Based on such findings, Gauthier and colleagues (Gauthier, Skudlarski, et al., 2000; Tarr \& Gauthier, 2000) have suggested that activation in the FFA reflects the influence of perceptual expertise on a flexible fusiform area that comes to respond robustly when participants have learned to discriminate the subtle perceptual differences that distinguish highly familiar items.

The contribution of the current work is to suggest that a process-based account of fusiform activation like that proposed by Gauthier can also explain category effects in tasks that do not require very specific categorization or identification of stimulus items. Intermediate-level classification of animals, like the specific identification of individual faces, requires the discrimination of similar visual and semantic representations. The intermediatelevel classification of artifacts does not make comparable demands, because artifacts with different intermediatelevel names do not have so many visual or semantic properties in common. Because fusiform responses are modulated by the differentiation demands of the task (Joseph \& Gathers, 2003), this structural difference in category representations yields a difference in fusiform activation for tasks involving intermediate-level classification of animals and artifacts. When the differentiation demands of the task are comparable, as in the specific condition of the current study, category effects are not observed. Similar arguments have been put forward in several recent articles (e.g., Joseph \& Gathers, 2003; Price et al., 2003), however, to our knowledge, the current work is the first to demonstrate robust domainlevel category effects that vanish when the differentiation demands of the task increase.

Our account of category-specific responding in the fusiform is also broadly consistent with a variety of computational models of visual-semantic processing. For example, in the cascade model of object naming from vision (Humphreys, Riddoch, \& Quinlan, 1988), visual stimuli activate localist visual representations (structural descriptions) of familiar items in proportion to their visual/structural similarity. Structural descriptions then compete for activation. For items that have many visually similar neighbors, the competition in this layer takes longer to resolve, producing "visual crowding" effects (Gale et al., 2001). Structural descriptions, in turn, project to localist semantic representations, which also compete for activation. Items in dense semantic neighborhoods have many competitors; hence, retrieval of semantic information specific to such items may suffer from a "semantic crowding" effect. Similar phenomena can also arise in models that adopt more distributed visual and/or semantic representations (Rogers et al.,
2004; Farah \& McClelland, 1991). Visual crowding has been shown to produce apparent category effects both in normal controls (Gaffan \& Heywood, 1993) and in patients with visual agnosia (Dixon, Bub, \& Arguin, 1997); semantic crowding effects produce different patterns of errors for animal and artifact items in patients with generalized semantic impairments (Rogers et al., 2004). Thus, apparent category-specific responding in the posterior fusiform could reflect either visual or semantic crowding effects, or both.

Many investigators have adopted the more general notion that knowledge about the properties of concrete objects is coded in a distributed and interactive cortical network, with many different regions contributing to the representation of a single object. This idea has a long history in cognitive neuropsychology-both Wernicke and Meynert articulated distributed theories of conceptual representation - and it has received widespread endorsement from recent functional imaging studies. Perhaps most notable in the current context are the network analyses of fMRI data conducted by Haxby et al. (2001). To answer the question of which regions in the ventral temporal cortex best predict category membership of stimulus objects, these authors presented participants with pictures of faces, animals, manmade objects, or nonsense photographs in a one-back repetition detection paradigm. The results showed that category membership was signaled not by the activation of discrete cortical modules for different kinds of objects but instead by the pattern of activity across many different regions. Although some areas appeared to be "selectively" active for some categories more than others, patterns of activation within these regions could still be used to predict category membership for the nonpreferred stimulus items; even when these "specialized" regions were excluded from the analysis, category membership could be reliably determined from the pattern of activity across other regions of cortex. From this analysis, Haxby et al. (2001) concluded that visual representations of object classes are coded in distributed and largely overlapping representations in the ventral temporal lobe.

We view Haxby's ideas as very similar in spirit to our own proposals about the representation of conceptual knowledge in cortex (e.g., Rogers et al., 2004; McClelland \& Rogers, 2003; Mummery et al., 1998; Vandenberghe, Price, Wise, Josephs, \& Frackowiak, 1996). Our data do, however, speak to one issue critical to any distributed theory of conceptual knowledge: What principles govern the neuroanatomical organization of the cortical semantic network? Most investigators would endorse an organization informed at least coarsely by perceptual modality, in which representations of object color are coded near regions responsible for color perception, representations of visual motion are coded near regions responsible for motion perception, and so on (Martin, Haxby, Lalonde, Wiggs, \& Ungerleider, 
1995). At issue is whether there exists further organization within a given modality, such that the properties that characterize different semantic categories are coded in separate subregions. Although the results of Chao et al. (1999) have generally been taken as supporting this idea, our data suggest that category effects in the lateral fusiform arise primarily from differentiation demands and do not reflect the anatomical organization of different kinds of visual features. On this view, visual representations for all different kinds of objects are coded in distributed patterns of activity across a network of shared regions in ventral temporal cortex, consistent with the ideas of Haxby et al. (2001), with the added caveat that some regions of the network are especially sensitive to perceptual or semantic differentiation demands of the particular task being performed.

As previously noted, animal-related activations are not the only category-specific patterns reported in the posterior fusiform. Chao et al. (1999) also described a region situated medially to the "animal" region, with differential activation for semantic tasks involving tools relative to animals. This finding is significant in the current context, because it appears to indicate a double dissociation of activity within the fusiform. Martin and Chao (2001) linked their finding to results indicating that the lateral-posterior fusiform responds more robustly to faces than to other objects (Kanwisher, McDermott, \& Chun, 1997), whereas a region situated more medially activates more strongly for buildings than for other objects (Aguirre, Zarahn, \& D'Esposito, 1998). From these results, together with their own data, the authors suggested a lateral-medial axis within the posterior fusiform, along which visual representations of animate objects are discerned from those of artifacts.

In the current experiment, however, we failed to find any region that was generally more active for artifacts than for animals. This may be because we used vehicles rather than tools as our artifact category-tools and vehicles likely differ in many of their visual, functional, and associative properties. Alternatively, the null result may reflect smoothing effects that arise in PET, although it should be noted that the lateral-medial double dissociation has been reported for faces and buildings in PET studies with the same scanner and analysis routines as used here (Gorno-Tempini \& Price, 2001). Other recent studies, however, have also failed to find artifact-related activity in the medial fusiform. Price et al. (2003) were unable to detect such an effect with PET, even using a very small smoothing kernel. In their meta-analysis of several imaging studies, these authors successfully replicated the finding of animal-related activity in the lateral fusiform but did not uncover artifact-related activity in the medial fusiform.

Taken together, the results suggest that categoryspecific double dissociations in different regions of the fusiform are not easily replicated. Reports of double dissociation are of great theoretical significance, how- ever, because they suggest that category-related activations cannot be explained solely with reference to a single process that is taxed more heavily in tasks requiring the fine discrimination of visual or semantic competitors. To account for category-specific double dissociations, process-based theories must posit multiple processes, which may be differentially involved in different semantic tasks and which may be taxed more heavily by some kinds of objects than others. It will, therefore, be important to determine whether the toolrelated activity in the medial fusiform, where it is replicated, persists for categorization at a specific level. In the mean time, we hypothesize that animal-related activity in the posterior fusiform is attributable to processing demands that result from representational similarity structure.

\section{METHODS}

\section{Subjects}

The task was piloted on 12 male subjects between the ages of 19 and 39 years (mean age 25 years) selected from the Cognition and Brain Sciences Unit volunteer panel. An additional 12 male subjects (same age range and mean) participated in the imaging study. All participants were right-handed native English speakers, free from any history of neurological disease or mental illness, and not on any medication. The study was approved by the local hospital ethics committee and the Administration of Radioactive Substances Advisory Committee (UK). All subjects in the imaging experiment gave written informed consent prior to receiving a PET scanning session consisting of 12 measurements.

\section{Stimuli}

Stimuli consisted of 48 color photographs of real animals and vehicles, including the following: 4 robins, 4 kingfishers, and 4 other birds; 4 Labradors, 4 Pekinese, and 4 other dogs; 4 BMWs, 4 Morris Travellers, and 4 other cars; and 4 ferries, 4 yachts, and 4 other boats. Examples of some of the photographs are shown in Figure 3. The items chosen for the specific categories (robin, kingfisher, Labrador, Pekinese, BMW, Morris, ferry, and yacht) were tested for name agreement in a separate behavioral study and were successfully named at the specific level by university undergraduates with greater than $72 \%$ agreement.

\section{Task and Design}

In each trial, participants viewed a category label (e.g., "dog") followed by a color photograph and were asked to indicate by button-press whether the photograph matched the word. Three different levels of specificity 


\section{Specific}

pekinese / labrador
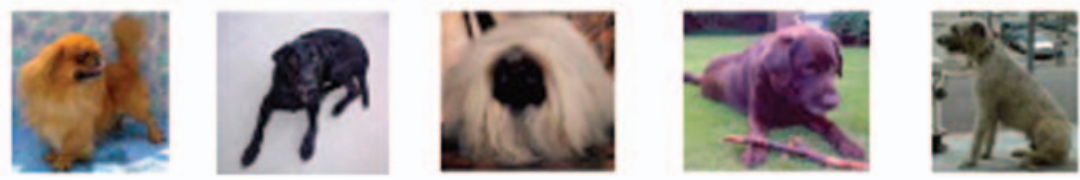

$\mathrm{BMW} /$ Morris
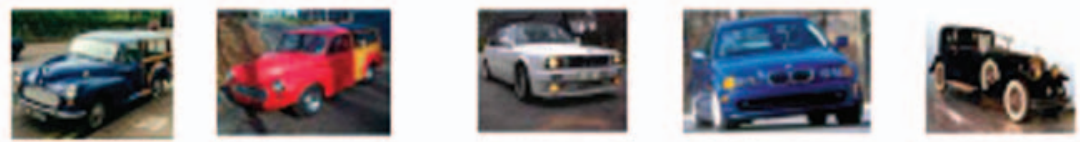

Intermediate

$\operatorname{dog} /$ bird
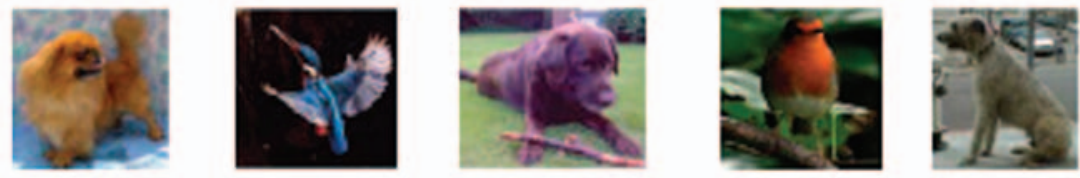

car / boat
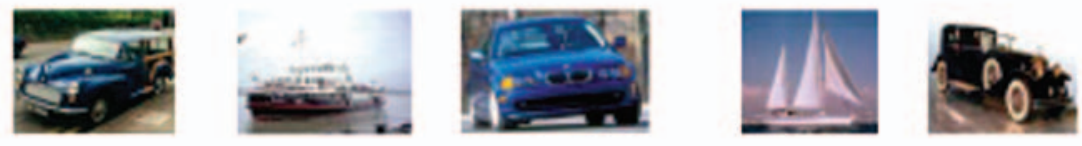

General

animal / vehicle
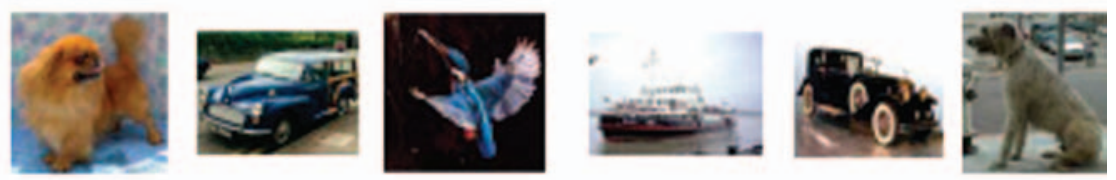

Figure 3. Examples of some of the stimuli used in the experiment. In the specific condition, all items within a scan were from the same category (e.g., different kinds of dogs, different kinds of car, etc.). In the intermediate condition, all items were from the same semantic domain (e.g., all animals or all vehicles). In the general condition, items from both domains were intermixed.

were used for the category labels: specific (e.g., Labrador, BMW), intermediate (e.g., dog, car) and general (e.g., animal, vehicle). Exactly the same set of 48 photographs were viewed in all three task conditions. Half of the trials were target trials, in which the photograph matched the word, and half were distracter trials. For specific trials, distracters were drawn from the same semantic category as the probe word (e.g., if the probe was "Labrador," the distracter was always a different breed of dog). For intermediate trials, the distracter was from a different category in the same superordinate domain (e.g., if the probe was "dog," the distracter was always a different kind of animal); for general-level trials, the distracter was always from the contrasting semantic domain (e.g., if the target was "animal," the distracter was always a vehicle).

Trials were blocked in a design manipulating semantic domain (animal or vehicle) and level of specificity (general, intermediate, and specific). The blocks were not fully crossed, because items from both domains were, of necessity, intermixed within block in the general condition, and PET procedures do not permit examination of the data in a stimulus-specific fashion. Thus, there were five experimental conditions in total: specific and intermediate categorization for animals and vehicles, plus the general categorization condition. This allowed us to investigate main effects of domain and specificity and their interaction for the intermediate and specific categorization tasks and also to compare these effects to responses generated in the general-level condition.

Each block (one PET scan in the imaging experiment) included 16 trials, half targets and half distracters. Two different category labels were used within each block, indicating two categories at the same level of specificity, ordered at random. For instance, in one specific block, participants might see the probes "Labrador" and "Pekinese" occurring in random order, and following each word, one of the 12 possible dog pictures would appear (with half the photographs matching the word and half not). Four items were repeated within block to make 16 items total. In an intermediate block, the participant might see the labels "bird" and "dog," with 8 of the possible dog pictures intermixed with 8 of the birds. In a general block, the labels "animal" and "vehicle" would appear, with 4 of the dog pictures intermixed with 4 each of the birds, boats, and cars. Thus, within each block, only two category labels ever appeared, and exactly 16 trials occurred, half targets and half distracters.

Following this design, the 48 items were arranged into four different blocks at each of the general, intermediate, and specific conditions, yielding 12 experimen- 
tal blocks in total, with the same 48 items appearing in all three task conditions. Because of the limitations of PET, it was impossible to administer all 12 experimental blocks as well as the necessary baseline blocks to every participant. Instead, experimental blocks in the PET experiment were assigned to participants in the following way: All participants completed all four specific blocks (one each for specific categorization of dogs, birds, cars, and boats); two of the four intermediate blocks (one each for intermediate categorization of animals and vehicles); and three of the four general blocks (with animals and vehicles intermixed). The assignment of intermediate- and general-level blocks was then counterbalanced across participants. Note that this design provided the greatest power to detect domain differences in the specific condition, and the least power to detect them in the intermediate condition (in which it was predicted that such effects would be observed). With one block equated to one scan during the PET procedure, this design yielded nine experimental scans per subject and ensured that all 48 photographs were viewed an equivalent number of times within each of the specific, intermediate, and general conditions across participants. In the behavioral pilot study, all participants completed all 12 blocks.

In addition to the nine experimental scans, each participant in the PET study also completed three baseline scans. In the baseline task, subjects viewed the word left or right, followed by a scrambled photograph, and were instructed to press the left or the right response button accordingly. Again, 16 trials appeared in each scan.

The only difference in procedure for the behavioral pilot and PET experiment concerned the duration with which stimulus events were presented. In both cases, the written category label (or the word indicating left or right in baseline scans) appeared on the screen for $750 \mathrm{msec}$ and was then replaced by the photograph or scrambled picture. In the behavioral pilot, the picture remained on screen until the participant responded, at which point it was replaced with a blank screen for $750 \mathrm{msec}$. In the PET study, each picture remained on screen for $1000 \mathrm{msec}$, and in turn, was replaced by a white screen until the start of the next trial $(2000 \mathrm{msec}$ later). Each trial in the PET study thus lasted precisely $3750 \mathrm{msec}$, and participants viewed each image for $1000 \mathrm{msec}$. In both the pilot and imaging study, participants viewed and named all of the 48 photographs once prior to the experiment, to reduce any effects of priming when the item was repeated. All participants were instructed to respond as quickly and accurately as possible.

\section{Positron Emission Tomography Scanning}

The 12 PET scans were obtained using a Siemens/CTI (model 962) PET scanner (Knoxville, TN). Each of the 12 scans involved a 20-sec intravenous bolus of $\mathrm{H}_{2}{ }^{15} \mathrm{O}$ at a concentration of $55 \mathrm{MBq} / \mathrm{ml}$ and a flow rate of $10 \mathrm{ml} / \mathrm{min}$ through a forearm cannula. For each subject, a T1weighted structural magnetic resonance image was also obtained with a 2-T Magnetom VISION scanner (Siemens, Erlangen, Germany).

\section{Data Analysis}

Behavioral data were analyzed in SPSS using repeated measures ANOVA to investigate RTs and error rates. The PET data were analyzed with statistical parametric mapping (SPM99, Wellcome Department of Imaging Neuroscience, London, UK, http://www.fil.ion.ucl.ac.uk/ spm) implemented in Matlab (Mathworks, Sherborn, MA) using standardized procedures (Friston, Worsley, Poline, Frith, \& Frackowiak, 1995). The mean image created by the realignment procedure was used to determine the parameters for transforming the images onto the Montreal Neurological Institute mean brain. These parameters were then applied to the functional images (Ashburner \& Friston, 1997) and the image was resampled into isotropic $2-\mathrm{mm}^{3}$ voxels. Finally, each image was smoothed with a 16-mm full-width halfmaximum Gaussian filter. The SPM software was then used to compute multiple linear regression analyses. To test regionally specific hypotheses, estimates were compared using linear compounds or contrasts. The resulting set of voxel values for each contrast is an SPM of the $T$ statistic that is converted into $Z$ scores.

\section{Acknowledgments}

This work was funded by a grant from the Wellcome Trust (Cathy Price, principal investigator). A. Mechelli was supported by National Institutes of Health (MH6445).

Reprint request should be sent to Timothy $\mathrm{T}$. Rogers, Department of Psychology, University of Wisconsin-Madison, 1202 W. Johnson Street, Madison, WI 53726, or via e-mail: ttrogers@wisc.edu; Cathy Price, Functional Imaging Laboratory, Wellcome Department of Imaging Neuroscience, 12 Queen Square, London WC1N 3BG, UK, or via e-mail: c.price@fil.ion.ucl.ac.uk.

\section{REFERENCES}

Aguirre, G. K., Zarahn, E., \& D’Esposito, M. (1998). An area within human ventral cortex sensitive to "building" stimuli: Evidence and implications. Neuron, 21, 373-383.

Ashburner, J., \& Friston, K. J. (1997). Multimodal image coregistration and partitioning: A unified framework. Neuroimage, 5, 271-279.

Bukach, C. M., Bub, D., Masson, M. E. J., \& Lindsay, D. S. (2004). Category specificity in normal episodic learning: Applications to object recognition and category-specific agnosia. Cognitive Psychology, 48, 1-46.

Caramazza, A. (1998). The interpretation of semantic category-specific deficits: What do they reveal about the 
organization of conceptual knowledge in the brain?

Neurocase, 4, 265-272.

Caramazza, A., \& Mahon, B. Z. (2003). The organization of conceptual knowledge: The evidence from category-specific semantic deficits. Trends in Cognitive Science, 7, 354-361.

Caramazza, A., \& Shelton, J. R. (1998). Domain-specific knowledge systems in the brain: The animate-inanimate distinction. Journal of Cognitive Neuroscience, 10, 1-34.

Chao, L. L., Haxby, J. V., \& Martin, A. (1999). Attribute-based neural substrates in temporal cortex for perceiving and knowing about objects. Nature Neuroscience, 2, 913-919.

Dixon, M., Bub, D., \& Arguin, M. (1997). The interaction of object form and object meaning in the identification performance of a patient with category-specific visual agnosia. Cognitive Neuropsychology, 14, 1085-1130.

Farah, M., \& McClelland, J. L. (1991). A computational model of semantic memory impairment: Modality-specificity and emergent category-specificity. Journal of Experimental Psychology: General, 120, 339-357.

Friston, K. J., Worsley, K. J., Poline, J. B., Frith, C. D., \& Frackowiak, R. S. J. (1995). Statistical parametric maps in functional imaging: A general linear approach. Human Brain Mapping, 2, 189-210.

Gaffan, D., \& Heywood, A. (1993). A spurious category-specific visual agnosia for living things in normal humans and nonhuman primates. Journal of Cognitive Neuroscience, 5, 118-128.

Gale, T. M., Done, D. J., \& Frank, R. J. (2001). Visual crowding and category specific deficits for pictorial stimuli: A neural network model. Cognitive Neuropsychology, 18, 509-550.

Garrard, P., Lambon Ralph, M. A., Hodges, J. R., \& Patterson, K. (2001). Prototypicality, distinctiveness and intercorrelation: Analyses of the semantic attributes of living and nonliving concepts. Cognitive Neuroscience, $18,125-174$

Gauthier, I. (2000). What constrains the organization of the ventral temporal cortex? Trends in Cognitive Science, 4, $1-2$.

Gauthier, I., Skudlarski, P., Gore, J. C., \& Anderson, A. W. (2000). Expertise for cars and birds recruits brain areas involved in face recognition. Nature Neuroscience, 3, 191-197.

Gauthier, I., Tarr, M. J., Anderson, A. W., Skudlarski, P., \& Gore, J. C. (1999). Activation of the middle fusiform "face area" increases with expertise in recognizing novel objects. Nature Neuroscience, 2, 568-573.

Gorno-Tempini, M. L., Cipolotti, L., \& Price, C. (2000). Category differences in brain activation studies: Where do they come from? Proceedings of the Royal Society of London, Series B: Biological Sciences, 267, 1253-1258.

Gorno-Tempini, M., \& Price, C. (2001). Identification of famous faces and buildings: A functional neuroimaging study of semantically unique items. Brain, 124, 2087-2097.

Grill-Spector, K., Knouf, N., \& Kanwisher, N. (2004). The fusiform face area subserves face perception, not generic within-category identification. Nature Neuroscience, 7 , 555-562.

Haxby, J. V., Gobbini, M. I., Furey, M. L., Ishai, A., Schouten, J. L., \& Pietrini, P. (2001). Distributed and overlapping representations of faces and objects in ventral temporal cortex. Science, 293, 2425-2430.
Humphreys, G. W., Riddoch, J., \& Price, C. (1997).

Top-down processes in object identification: Evidence from experimental psychology, neuropsychology and functional anatomy. Philosophical Transactions of the Royal Society of London B, 352, 1275-1282.

Humphreys, G. W., Riddoch, M. J., \& Quinlan, P. T. (1988). Cascade processes in picture identification. Cognitive Neuropsychology, 5, 67-103.

Joseph, J. E. (2001). Functional neuroimaging studies of category specificity in object recognition: A critical review and meta-analysis. Cognitive, Affective and Behavioral Neuroscience, 1, 119-136.

Joseph, J. E., \& Gathers, A. D. (2003). Effects of structural similarity on neural substrates for object recognition. Cognitive, Affective and Behavioral Neuroscience, 3, 1-16.

Kanwisher, N. (2000). Domain specificity in face perception. Nature Neuroscience, 3, 759-763.

Kanwisher, N., McDermott, J., \& Chun, M. M. (1997). The fusiform face area: A module in human extrastriate cortex specialized for the perception of faces. Journal of Neuroscience, 17, 4302-4311.

Kanwisher, N., Stanley, D., \& Harris, A. (1999). The fusiform face area is selective for faces, not animals. NeuroReport, 10, 183-187.

Malach, R., Levy, I., \& Hasson, U. (2002). The topography of high-order human object areas. Trends in Cognitive Science, 6, 176-184.

Martin, A., \& Chao, L. L. (2001). Semantic memory in the brain: Structure and processes. Current Opinion in Neurobiology, 11, 194-201.

Martin, A., Haxby, J. V., Lalonde, F. M., Wiggs, C. L., \& Ungerleider, L. G. (1995). Discrete cortical regions associated with knowledge of color and knowledge of action. Science, 270, 102-105.

McClelland, J. L., \& Rogers, T. T. (2003). The Parallel Distributed Processing approach to semantic cognition. Nature Reviews Neuroscience, 4, 310-322.

McRae, K., \& Cree, G. (2002). Factors underlying category-specific deficits. In E. M. E. Forde, \& G. Humphreys (Eds.), Category specificity in brain and mind. Hove: Psychology Press.

Monsell, S., Doyle, M. C., \& Haggard, P. N. (1989). Effects of frequency on visual word recognition tasks: Where are they? Journal of Experimental Psychology: General, 118, 43-71.

Moore, C., \& Price, C. (1999). A functional neuroimaging study of the variables that generate category-specific object processing differences. Brain, 122, 943-962.

Mummery, C. J., Patterson, K., Hodges, J. R., \& Price, C. J. (1998). Functional neuroanatomy of the semantic system: Divisible by what? Journal of Cognitive Neuroscience, 10, 766-777.

Price, C. J., Noppeney, U., Phillips, J., \& Devlin, J. T. (2003). How is the fusiform gyrus related to category-specificity? Cognitive Neuropsychology, 20, 561-574.

Rogers, T. T., Lambon Ralph, M. A., Garrard, P., Bozeat, S., McClelland, J. L., Hodges, J. R., \& Patterson, K. (2004). The structure and deterioration of semantic memory: A computational and neuropsychological investigation. Psychological Review, 111, 205-235.

Rosch, E., Mervis, C. B., Gray, W., Johnson, D., \& Boyes-Braem, P. (1976). Basic objects in natural categories. Cognitive Psychology, 8, 382-439.

Talairach, J., \& Tourneaux, P. (1988). Co-planar stereotaxic atlas of the buman brain. New York: Thieme. 
Tarr, M. J., \& Gauthier, I. (2000). FFA: A flexible fusiform area for subordinate-level visual processing automatized by expertise. Nature Neuroscience, 3, 764-769.

Thompson-Schill, S. L. (2003). Neuroimaging studies of semantic memory: Inferring "how" from "where." Neuropsychologia, 41, 280-292.

Tversky, B., \& Hemenway, K. (1984). Objects, parts, and categories. Journal of Experimental Psychology, 113, 169-191.

Tyler, L. K., \& Moss, H. E. (2001). Towards a distributed account of conceptual knowledge. Trends in Cognitive Sciences, 5, 244-252.

Tyler, L., Moss, H. E., Durrant-Peatfield, M. R., \& Levy, J. P. (2000). Conceptual structure and the structure of concepts: A distributed account of category-specific deficits. Brain and Language, 75, 195-231.

Vandenberghe, R., Price, C., Wise, R., Josephs, O., \& Frackowiak, R. S. J. (1996). Functional anatomy of a common semantic system for words and pictures. Nature, 383, 254-255. 